\title{
Physique atomique et moléculaire, 1973-2004
}

\section{Claude Cohen-Tannoudji}

\section{(2) OpenEdition}

\section{Journals}

Édition électronique

URL : https://journals.openedition.org/annuaire-cdf/1067

DOI : 10.4000/annuaire-cdf. 1067

ISBN : 978-2-7226-0325-7

ISSN : 2109-9227

Éditeur

Collège de France

Édition imprimée

Date de publication : 1 avril 2013

Pagination : 776-778

ISBN : 978-2-7226-0198-7

ISSN : 0069-5580

\section{Référence électronique}

Claude Cohen-Tannoudji, « Physique atomique et moléculaire, 1973-2004 », L'annuaire du Collège de France [En ligne], 112 | 2013, mis en ligne le 22 novembre 2013, consulté le 22 août 2022. URL : http:// journals.openedition.org/annuaire-cdf/1067 ; DOI : https://doi.org/10.4000/annuaire-cdf.1067 
Memorial Symposium for Vivian Teichberg : «Allosteric properties of ligand-gated ion channels : lessons from prokaryotic receptors », Weizmann Institute Rehovot, Israël, 2 mai 2012.

Conférence : «Genetic and epigenetic constraints on cognitive development », colloque «Variance \& Invariants in Brain Behavior», organisé par Technion Institute of Technology à Haifa, Israël, 21-23 mai 2012.

Conférence : «Genetic and epigenetic constraints on cognitive development », workshop : « Core Knowledge, language and Culture », à Lorentz Center, Leiden, Pays-Bas, du 28 mai au 3 juin 2012.

Conférence : «The Pharmaceutical Colloquium lecture series », à l'Université de Bonn : «Allosteric mechanisms of signal transduction in neurotransmitter receptors : implications for drug design », à Bonn, Allemagne, 2 juillet 2012.

\section{Claude COHEN-TANNOUDJI, membre de l'Institut (Académie des sciences)}

\section{Physique atomique et moléculaire, 1973-2004}

\section{PuBlications}

Wolf P., Blanchet L., Bordé C.J., Reynaud S., Salomon C. et Cohen-Tannoudji C., « Reply to the comment on 'Does an atom interferometer test the gravitational redshift at the Compton frequency?' », Class. Quantum Grav., 29, 2012, 048002.

Wassen W., Cohen-Tannoudji C., Leduc M., Boiron D., Westbrook C.I., Truscott A., Baldwin K., Birkl G., Cancio P., Trippenbach M., «Cold and trapped metastable noble gases », Rev. Mod. Phys., 84, 2012, 175.

\section{Ouvrages}

Cohen-Tannoudji C. et Guéry-Odelin D., «Advances in Atomic Physics - An overview », World Scientific, Singapour, 2011, ouvrage de 750 p.

\section{CONFÉRENCES}

(Invitations à des conférences internationales, conférences spéciales et commémoratives, conférences grand public)

Science and Innovation Week (SIW 2011), série de trois conférences, Mexique, 17, 19 et 21 octobre 2011: «Advances in Atomic Physics. From Optical Pumping to Ultracold Atoms », «Laser Manipulation of Atoms. From Basic Research to Applications ».

Congrès annuel de l'Union des professeurs de physique et de chimie 2011, Université de Montpellier 2, 24 octobre 2011: «Atomes et photons : de la recherche fondamentale aux applications ». 
Honeywell Initiative for Science and Engineering, Pékin, Chine, 15 novembre 2011, conférence générale : "Laser Manipulation of Atoms », colloque : "Atomic Clocks with Ultracold Atoms».

Marie-Curie-Symposium on the Physical Foundations of Modern Chemistry, Varsovie, Pologne, 19 novembre 2011: «Advances in Atomic Physics. From Optical Pumping to Ultracold Atoms and Molecules ».

Table ronde et colloque «Science Frontiers », Donostia International Physics Center, San Sebastian, Espagne, 2 décembre 2011.

Conférence de Malte $\mathrm{V}$, « Frontières de la science et de la chimie : recherche et éducation au Moyen-Orient », UNESCO, Paris, 6 décembre 2011 : « Measuring Time with Ultracold Atoms ».

France - Hong Kong Distinguished Lecture, City University of Hong Kong, Chine, 13 janvier 2012 : «Advances in Atomic Physics: From Optical Pumping to Quantum Gases ».

International Science Youth Forum 2012, «Nurturing the Scientific Mind », Institute of Advances Studies, Singapour :

- Nobel Laureate Master Class, 16 janvier 2012 : «Wave-Particle Duality. From Light to Matter»;

- Nobel Laureate Master Class, 17 janvier 2012 : «Manipulating Atoms with Light»;

- Conférence publique, National University of Singapore, 20 janvier 2012: «Advances in Atomic Physics: From Optical Pumping to Ultracold Atoms ».

Norman Ramsey Memorial Service, Université de Harvard, Boston, États-Unis, 3 mars 2012.

National Tsing Hua University, Hsinchu, Taiwan, Chine :

- 21 mai 2012: «Advances in Atomic Physics. From Optical Pumping to Quantum Gases $»$;

- 22 mai 2012: «Light and Matter».

Photonics without Frontiers, EPFL Research Day, École polytechnique fédérale de Lausanne, Lausanne, Suisse, 14 juin 2012 : « Atoms and Light ».

Assosciences Midi-Pyrénées, Toulouse, France, 19 juin 2012 : «Atomes et lumière »

Croisière «Les grands récits de l'Univers. Science, religion, philosophie : le monde est-il compréhensible? », série de deux conférences du 6 au 18 juillet 2012 : «Une brève histoire de la lumière et de la matière ».

\section{RESPONSABILITÉS DIVERSES}

Président de la fondation Paris Sciences et Lettres - Quartier Latin, jusqu' au 8 février 2012. Président d'honneur du conseil scientifique de la Fondation France-Israël.

Président du Comité de défense des hommes de science (CODHOS) de l'Académie des sciences.

Membre du conseil exécutif de l'International Human Rights Network of Academies and Scholarly Societies (IHRNASS).

Membre du conseil scientifique de la Fondation européenne pour les énergies de demain (FEED).

Membre du comité pour le prix international pour l'alphabétisation scientifique des enfants de la planète.

Membre du comité de pilotage "Science à l'école » depuis 2004.

Membre du conseil scientifique de l'Institut francilien de recherche sur les atomes froids (IFRAF) depuis 2004.

Membre du conseil scientifique de l'Israëli-Palestinian Science Organisation (IPSO). 


\title{
DISTINCTIONS
}

Honorary Professor de l'Université d'aéronautique et d'astronautique de Pékin (BUAA) Université BeiHang, Pékin, Chine, 2011.

\section{Yves Coppens, membre de l'Institut (Académie des sciences)}

\section{Paléoanthropologie et préhistoire, 1983-2005}

\author{
PUBLICATIONS (JUIN 2011-JUIN 2012)
}

Coppens Y., Préface, in London J., Avant Adam ; format numérique, Libella, octobre 2011.

Coppens Y., Leçon inaugurale au Collège de France [1983] : réédition électronique, Collège de France.

Coppens Y., Préface in Laporte G., Voyage au pays du temps qui court, édition À nos pages, 2011, 11-15.

Coppens Y., Préface in L'identité retrouvée, Les reconstructions anatomiques d'Elisabeth Daynès, Musée départemental de préhistoire d'Ile de France, 2011, 10.

Coppens Y., Préface in Abécédaire de l'innovation, Université de technologie de Compiègne, 2011, 7-11 ; édition anglaise, 2012.

Coppens Y., Préface in Fourny P., Homo erectus, le combat d'une profession, Le ChercheMidi, 2011, 8-11.

Coppens Y., Avant-propos in Delaine J., Heckendorn A. et Roth-Zehner M. (dir), Trésors d'Archéologie, Éditions JdM, Mulhouse, 2011, 11-13.

Coppens Y., Préface in Grosjean F., Roger Grosjean, itinéraires d'un archéologue, Éditions Alain Piazzola, 2011, 11-12.

Coppens Y., «Un génie, produit d'un changement climatique », Sciences et Avenir, Horssérie, «Qu'est-ce que l'Homme, 100 scientifiques répondent », janvier-février 2012, 14.

Coppens Y., Le journal de votre croisière, Le Princess Danae, croisière Groenland, TerreNeuve, Canada, août 2011.

Coppens Y., Préface in Pelot P., Sous le Vent du Monde, Éditions Omnibus, 2012, 9-11, 251-253, 515-516, 763-764, 1065-1066 (anthologie de 5 romans épiques, Qui regarde la montagne au loin, Le nom perdu du soleil, Debout dans le ventre blanc du silence, Avant la fin du ciel, Ceux qui parlent au bord de la pierre, conseils scientifiques d'Yves Coppens, 1247 p.).

Coppens Y., «La diversité biologique depuis 10 millions d'années et l'émergence de l'homme » in de Lumley H. (dir.), l'Univers, la Vie, l'Homme, émergence de la conscience, CNRS éditions, 2012, 118-121.

Patou-Mathis M. et Coppens Y., «Aux origines de la pluralité humaine » in Thuram L., Manifeste pour l'égalité, Autrement, 2012, 48-55.

Coppens Y., Avant-propos, «L'Homme est un produit de l'environnement », in Berger J.F. (dir.), Des climats et des hommes, La Découverte, 2012, 11-16. 